Pacific Journal of Mathematics

ENTIRE FUNCTIONS 


\section{ENTIRE FUNCTIONS}

\section{A. C. Schaeffer}

1. Introduction. The object of this note is to prove several results, which are related in that each is concerned with entire functions of exponential type. An entire function $f(z)$ is of exponential type $\tau$ if for every $\varepsilon>0$ there is a number $A=A(\varepsilon)$ such that

$$
|f(z)| \leqq A e^{(\tau+\varepsilon)|z|} .
$$

The function is of precise type $\tau$ if (1) does not hold for any $\varepsilon<0$.

The first result is concerned with entire functions which are bounded at a sequence of points. Miss Cartwright's theorem states that if $f(z)$ is an entire function of exponential type $\tau, \tau<\pi$, and is bounded by 1 at the integer points,

$$
|f(n)| \leqq 1, \quad n=0, \pm 1, \pm 2, \cdots,
$$

then $f(z)$ is bounded on the entire real axis by a number which depends only on $\tau$,

$$
|f(x)|<M(\tau), \quad-\infty<x<\infty .
$$

Proofs of this and stronger results have been given by Cartwright, Pflunger, Macintyre, Boas, Korevaar, Duffin and Schaeffer, Levin, Ahiezer, Agmon, and others. These results are discussed in [2, Chapter 10] where further references are given.

Let $N$ be a sequence of integers. The first question to be considered in the present note is : what conditions must $N$ satisfy in order that for every entire function of exponential type less than $\pi$ the condition

$$
|f(n)| \leqq 1,
$$

$n \in N$,

will imply that $f(z)$ is bounded on the real axis? To answer this question we define a function $\lambda(t)$ for $t>0$ by means of the given sequence $N$. Let $\lambda(t)$ be the greatest integer $\mu$ such that every interval of the real axis of length $t$ has $\mu$ or more elements of $N$. For any positive $t$ there is at least one interval, which may be supposed open, of length $t$ which contains precisely $\lambda(t)$ elements of $N$, and every interval of length $t$ whether open or closed contains $\lambda(t)$ or more elements of $N$. The following result is to be proved.

Received March 1, 1955. This paper represents work done under contract N7 onr28507 with the Office of Naval Research. 
THEOREM 1. If $N$ is a sequence of integers, the condition

$$
\lim _{t \rightarrow \infty} \frac{\lambda(t)}{t}=1
$$

is necessary and sufficient in order that every entire function of exponential type less than $\pi$ which is bounded on $N$ be bounded on the real axis.

Slightly more is true than stated in the theorem, for there is a uniform bound in the following sense. If (5) is true then any entire function $f(z)$ of exponential type $\tau, \tau<\pi$, which satisfies (4) must also satisfy

$$
|f(x+i y)| \leqq M(\tau, N) e^{\tau|y|} .
$$

Thus the bound for $|f(x)|$ depends only on $N$ and the exponential type of $f$. Relation (6) also gives a dominant for $f(z)$ over the entire plane. ${ }^{1}$ As $\tau$ approaches $\pi$ the value of $M(\tau, N)$ must approach infinity. This was shown by Boas and the author, [2] where further references are given, in case $N$ consists of all the integers, and is a fortiori true for any sequence of integers.

The remaining topics to be considered in the present work center around research problem number 4 in the Bulletin of the American Mathematical Society [3]. This problem, which is due to Boas, reads as follows.

"Let $f(z)$ be an entire function of exponential type. It is well known, and easily proved by Phragmen-Lindelöf arguments, that if $f(x)$ is bounded or approaches a limit as $x \rightarrow \infty$, then $f(x+i y)$ is bounded or approaches a limit, for each $y$, as $x \rightarrow \infty$. If $|f(x)|$ approaches a nonzero limit, does $|f(x+i y)|$ necessarily approach a limit?" To answer this question an entire function $f(z)$ of exponential type will be defined such that

$$
\lim _{x \rightarrow \infty}|f(x)|
$$

exists and is finite, but

$$
\lim _{x \rightarrow \infty}|f(x+i y)|
$$

exists only for $y=0$.

In view of this example it is natural to ask what hypothesis in addition to (7) will imply that (8) exists for all $y$. It is to be shown that if (7) exists both for $f$ and one of its derivatives then (8) exists

1 A similar dominant was obtained by Miss Cartwright which strengthens inequality (3). 
for $f^{*}$ and all its derivatives. More precisely, we have the following theorem where $f^{(\nu)}(z)$ denotes the derivative of $f(z)$ of order v, with $f(z)=f^{(0)}(z)$.

THEOREM 2. If $f(z)$ is an entire function of exponential type such that for some $m, m \geq 1$, both $|f(x)|$ and $\left|f^{*(m)}(x)\right|$ tend to finite limits as $x \rightarrow \infty$ then

$$
\lim _{x \rightarrow \infty}\left|f^{(\nu)}(x+i y)\right|
$$

exists and is finite for all $y$ and for $\nu=0,1,2, \cdots$.

2. Proof of Theorem 1. The proof of Theorem 1 will depend in part on the following result of R. J. Duffin and the author [1], [4], and [2] where further references are given.

Theorem 3. Let $\left\{\lambda_{n}\right\}, n=0, \pm 1, \pm 2, \cdots$, be a sequence such that

$$
\begin{aligned}
& \left|\lambda_{n}-\lambda_{m}\right| \geqq \delta>0, \quad n \neq m, \\
& \left|\lambda_{n}-n\right| \leqq A
\end{aligned}
$$

for some $\delta, A$. If $f(z)$ is an entire function of exponential type $\tau$, $\tau<\pi$, such that

$$
\left|f\left(\lambda_{n}\right)\right| \leq 1
$$

then

$$
|f(x+i y)| \leq R e^{\tau|y|}
$$

where $R$ depends exclusively on $\tau, \delta, A$.

It is first to be shown that (4), (5) imply the boundedness of $f(x)$ for any entire function of exponential type less than $\pi$. Thus suppose that $N$ is a sequence of integers for which (5) is true, and consider any fixed number $\tau$ in the range $0 \leqq \tau<\pi$. If $f(z)$ is an entire function of exponential type $\tau$ which satisfies (4) and

$$
\alpha=\begin{aligned}
& \pi-\tau \\
& \pi+\tau
\end{aligned}
$$

then

$$
F(z)=f\left(\begin{array}{c}
z \\
1-\alpha
\end{array}\right)
$$

is an entire function of exponential type $\tau^{\prime}$.

$$
\tau^{\prime}=\begin{gathered}
\pi+\tau \\
2
\end{gathered}
$$

Now $F(z)$ is bounded by 1 at a sequence of points $\left\{\mu_{n}\right\}$ defined for 
$n \in N$, where

$$
\mu_{n}=n(1-\alpha),
$$

$n \in N$.

Using relation (5) it then follows that if $T$ is sufficiently large, each interval of the real axis of length $T$ contains at least $T$ element of $\left\{\mu_{n}\right\}$. Let $T$ be an integer. In each of the intervals

$$
\nu T \leqq x<(\nu+1) T
$$

choose $T$ elements of $\left\{\mu_{n}\right\}$ to form a new sequence $\left\{\lambda_{m}\right\}$. Then $\left\{\lambda_{m}\right\}$ is defined for $m=0, \pm 1, \pm 2, \cdots$, and

$$
\begin{array}{ll}
\left|\lambda_{m}-m\right|<T, & m=0, \pm 0, \pm 2, \cdots, \\
\left|\lambda_{m}-\lambda_{n}\right| \geq 1-\delta, & m \neq n .
\end{array}
$$

Since $\tau^{\prime}<\pi$, Theorem 3 shows that $F(z)$ is bounded on the real axis, and indeed

$$
|F(x+i y)| \leqq R e^{\tau^{\prime}|y|} .
$$

This proves that condition (5) is sufficient in Theorem 1 , and since the sequence $\left\{\lambda_{m}\right\}$ depends only on $\tau, N$ we see that $R$ is a function of $\tau, N$ only, which gives inequality (6).

In the proof of the necessity of condition (5) in Theorem 1 the following lemma will be used.

Lemma. If $h$ is an integer, $h \geq 5$, and in the closed interval $-h \leqq x \leqq h$ there is a set $N^{\prime}$ of integers whose number is $\rho, \rho \geq 2$, then there is an entire function $F(z)$ of exponential type $\mu$,

$$
\mu=\pi-\begin{gathered}
\pi \rho \\
32 h
\end{gathered},
$$

whose maximum modulus on the real axis occurs at some point $x_{0}$ satisfying $\left|x_{0}\right|<4 h+1$, and $F\left(x_{0}\right)=1$, but

$$
\begin{array}{ll}
|F(x)| \leqq 2^{1-\rho}, & |x| \geqq 14 h-1, \\
|F(n)| \leqq 2^{1-\rho}, & n \notin N^{\prime} .
\end{array}
$$

Proof. Since the object is to define a function $F(z)$ which is of exponential type $\mu$ and is small on the integer points except at the set $N^{\prime}$ we begin with the function $\sin \pi z$, which is of exponential type $\pi$ and vanishes at all the integers.

If $n_{1}, n_{2}, \cdots, n_{\alpha}$ are integers which belong to $N^{\prime}$ then the function

$$
f_{1}(z)=\frac{\sin \pi z}{\prod_{\nu=1}^{\infty} \sin \frac{\pi\left(z-n_{\nu}\right)}{16 h}}
$$


is of exponential type

$$
\mu^{\prime}=\pi-\frac{\alpha \pi}{16 h} .
$$

However, this function satisfies the functional relation $f_{1}(x+16 h)=$ $\pm f_{1}(x)$ so it attains its maximum modulus over the real axis at points is each interval of length $16 h$. If $m_{1}, m_{2}, \cdots, m_{\beta}$ are the remaining integers which belong to $N^{\prime}$ then the function

$$
f_{2}(z)=\frac{\sin \pi z}{\prod_{\nu=1}^{\beta}\left(z-m_{\nu}\right) \prod_{\nu=1}^{\alpha} \sin \frac{\pi}{16 h}\left(z-n_{\nu}\right)}
$$

remains of exponental type $\mu^{\prime}$ where $\mu^{\prime}$ is defined by (11), but it is small when $|x|$ is large. The function $f_{2}(z)$ vanishes at all integers in the range $|x| \leqq 15 h-1$ except those that belong to the set $N^{\prime}$. It is to be shown that if $\alpha, \beta$ are suitably chosen then a suitable constant times the function $f_{2}(z)$ is the function $F(z)$ whose existence is asserted in the lemma.

Let $\alpha=\rho / 2$ if $\rho$ is even and $\alpha=(\rho+1) / 2$ if $\rho$ is odd; and define

$$
\begin{aligned}
& \phi_{1}(z)=\prod_{\nu=1}^{\infty} \sin \frac{\pi}{16 h}\left(z-n_{\nu}\right) \\
& \phi_{2}(z)=\prod_{\nu=1}^{\beta}\left(z-m_{\nu}\right) .
\end{aligned}
$$

Here $m_{y}, n$, are together all the integers which belong to $N^{\prime}$ so we have

$$
\beta=\rho-\alpha \geqq \frac{\rho-1}{2} .
$$

By considering the individual terms of $\phi_{1}^{\prime}(x)$ separately it is clear that

$$
\left|\phi_{1}(1 / 2)\right|<\left|\phi_{1}(x)\right|, \quad 2 h+1 \leqq|x| \leqq 14 h-1 .
$$

The function $f_{1}(z)$ defined by (10) may be written

$$
f_{1}(z)=\begin{gathered}
\sin \pi z \\
\phi_{1}(z)
\end{gathered},
$$

and it therefore satisfies the inequality

$$
\left|f_{1}(1 / 2)\right|>\left|f_{1}(x)\right|, \quad 2 h+1 \leqq|x| \leq 14 h-1 .
$$

Since $\left|f_{1}(x)\right|$ is periodic with period $16 h$ the maximum modulus of $f_{1}(x)$ on the real axis is attained at some point $x_{1}$ in the interval $\left|x_{1}\right| \leq 8 h$. But then (13) shows that

$$
\left|x_{1}\right|<2 h+1 .
$$


Also, $f_{1}(z)$ is of exponential type $\mu^{\prime}$, and since $\alpha \geq \rho / 2$ we have $\mu^{\prime} \leq \mu^{\prime}$.

The function $f_{2}(z)$ defined by (12) may be written

$$
f_{2}(z)=\begin{aligned}
& f_{1}(z) \\
& \phi_{2}(z)
\end{aligned} .
$$

Now $f_{2}(x)$ attains its maximum modulus on the real axis at some point $x_{0}$ satisfying $\left|x_{0}\right|<4 h+1$. For if $|x| \geqq 4 h+1$ then using (14), we have

$$
\left|\phi_{2}(x)\right| \geqq(3 h+1)^{\beta}>\left|\phi_{2}\left(x_{1}\right)\right| .
$$

Hence

$$
\left|f_{2}(x)\right|=\left|\begin{array}{l}
f_{1}(x) \\
\phi_{2}(x)
\end{array}\right|<\left|f_{1}(x)\right| \leq\left|f_{2}\left(x_{1}\right)\right|, \quad|x| \geqslant 4 h+1
$$

We also have

$$
\left|\phi_{2}(x)\right| \geqq 4^{\beta}(3 h+1)^{\beta}>4^{\beta}\left|\phi_{2}\left(x_{1}\right)\right|, \quad|x| \geqq 14 h-1,
$$

where we have used the supposition $h \geq 5$. Thus for $|x| \geq 14 h-1$ we have

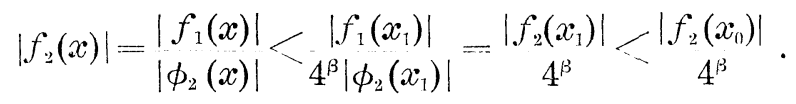

Since $\beta \geq(\beta-1) / 2$ the function

$$
F(z)=\begin{aligned}
& f_{2}(z) \\
& f_{2}\left(x_{0}\right)
\end{aligned}
$$

has all the properties stated in the lemma.

We now complete the proof of Theorem 1 by showing that condition (5) is necessary. It follows from the definition of $\lambda(t)$ that for any sequence of integers we automatically have

$$
\limsup _{t \rightarrow \infty} \lambda(t) \leq 1
$$

Thus suppose $N$ is a sequence of integers such that

$$
\liminf _{t \rightarrow \infty} \begin{gathered}
\lambda(t) \\
t
\end{gathered}=\gamma<1
$$

It is to be shown under this supposition that there is an entire function $G(z)$ of exponential type less than $\pi$ which is bounded on $N$, but not on the real axis. For this purpose the first objective is naturally to find arbitrarily long intervals $[a-h, a+h]$ in which we can use translations $F_{h}(z-a)$ of the functions defined in the lemma. The required function $G(z)$ will then be a suitable linear combination $\sum c_{n} F_{n}$ of these functions. 
Let

$$
\gamma_{1}=\frac{1+\gamma}{2}
$$

If $h_{0}$ is sufficiently large the following statement is true. Given a positive number $r$ and a positive interger $h, h \geq h_{0}$, there is a closed interval $I$ of length $2 h$ lying entirely in $|x|>r$ and with integer endpoints such that the number of elements of $N$ lying in $I$ is less than

$$
2 h \gamma_{1} \text {. }
$$

This can be proved by means of (15). The number of integer points in $I$ which do not belong to $N$ is therefore greater than

$$
2 h+1-2 h \gamma_{1} \text {. }
$$

Let $H$ be an integer such that $H \geq h_{0}, H \geq 5$; and, for later purposes, we also choose $H$ so large that

$$
\sum_{h=H}^{\infty} h 4^{-h\left(1-\gamma_{1}\right)}<1
$$

For each integer $h$ satisfying $h>H$ there is a closed interval $I_{h}$ of length $2 h$ with integer end-points and center at $x_{l}$ where

$$
\left|x_{h+1}\right|>\left|x_{h}\right|+28 h+14, \quad h>H,
$$

and the number of integer points in $I_{l}$ which do not belong to $N$ is $\rho_{h}$, where by (16),

$$
\rho_{h}>2 h\left(1-\gamma_{1}\right)+1
$$

Take

$$
\left|x_{H}\right|>28 H
$$

We now use the lemma, where we can clearly translate the interval. The set $N^{\prime}$ of the lemma is the set of integers in $I_{h}$ which do not belong to $N$. There is an entire function $F_{h}(z)$ of exponential type $\lambda$,

$$
\lambda=\pi-\frac{\pi\left(1-\gamma_{1}\right)}{16}
$$

which satisfies

$$
\left|F_{h}^{\prime}(x)\right| \leqq 4^{-h\left(1-\gamma_{1}\right)}, \quad\left|x-x_{h}\right| \geqq 14 h-1 .
$$

In the last inequality we have made use of (19). Also,

$$
\left|F_{h}(n)\right| \leqq 4^{-n\left(1-\gamma_{1}\right)}
$$


We note also that because of (18) the intervals $\left|x-x_{h}\right| \leq 4 h-1$ are disjoint; hence at each point $x$ of the real axis the inequality

$$
\left|F_{h}(x)\right| \leq 4^{-n\left(1-\gamma_{1}\right)}
$$

is satisfied except possibly for one value of $h$. Each function $F_{h}^{\prime}(x)$ has maximum modulus 1 on the real axis, and this maximum is attained at some point $x_{h}^{\prime}$ satisfying $\left|x_{h}^{\prime}-x_{h}\right|<4 h+1$.

How consider the functions

$$
G_{m}(z)=\sum_{h=11}^{m} h F_{h}^{\prime}(z), \quad m=H, H+1, \cdots .
$$

If $\nu$ lies in the range $H \leqq \nu \leqq m$ then by (21),

$$
\left|G_{m}\left(x_{\nu}^{\prime}\right)\right| \geq \nu-\sum_{h \neq \nu} h\left|F_{h}\left(x_{\nu}^{\prime}\right)\right|>\nu-\sum_{h=H}^{\infty} h 4^{-n\left(1-\gamma_{1}\right)},
$$

so (17) yields

$$
\left|G_{m}\left(x_{\nu}^{\prime}\right)\right|>\nu-1
$$

To obtain a dominant for $G_{m}(z)$ on the real axis which is independent of $m$ we note first that if $x$ lies outside all the intervals $\left|x-x_{h}\right| \leq 14 h-1$ then (23) is valid for all $h$, and

$$
\left|G_{m}(x)\right|<1 \text {. }
$$

If, on the other hand, $x$ lies in one of these intervals, say $\left|x-x_{k}\right|<14 k-1$, then we shall have

$$
\left|G_{m}(x)\right|<k+\sum_{h=H}^{\infty} h 4^{-h\left(1-\gamma_{1}\right)<k+1} .
$$

But (18), (20) imply that $\left|x_{h}\right|>28 h$ for all $h$, so the inequality $\left|x-x_{k}\right|<14 k-1$ shows $|x|>14 k+1$. Thus

$$
\left|G_{m}(x)\right| \leq 1+\begin{gathered}
|x| \\
14
\end{gathered}
$$

Now $G_{m}(z)$ is an entire function of exponential type $\lambda$, so a dominant which is independent of $m$ can be obtained for $G_{m}(z)$ over the entire plane by use of (25). One argument makes use of Theorem 3 . The function $\left\{G_{m}(z)-G_{m}(0)\right\} / z$ is bounded by 3 on the part of the real axis for which $|x| \geqq 1$, and it is an entire function of exponential type $\lambda, \lambda<\pi$. Thus the function is bounded by 3 at a sequence $\left\{\lambda_{n}\right\}$ which satisfies the conditions of Theorem 3. Hence

$$
\left.\mid r_{r_{m}}(x+i \eta)\right)-G_{m}(0)\left|<R^{\prime}\right| z \mid e^{\lambda|\eta|}
$$

where $R^{\prime}$ is independent of $m$. 
Since $\left|G_{m}(0)\right| \leqq 1$ the functions $G_{m}(z), m=H, H+1, \cdots$, are uniformly bounded in each bounded domain, hence for some subsequence of $m$ tending to infinity the functions $G_{m}(z)$ tend to a limit $G(z)$. Moreover

$$
\left|G(x+i y) \leqq 1+R^{\prime}\right| z \mid e^{\lambda|y|}
$$

so $G(z)$ is an entire function of exponential type $\lambda, \lambda<\pi$. Inequalities (22), (17) show that

$$
|G(n)| \leqq 1
$$
$n \in N$,

but inequality (24) shows that

$$
\left|G\left(x_{\nu}^{\prime}\right)\right| \geqq \nu-1, \quad \nu=H, H+1, \cdots,
$$

and $G(z)$ is not bounded on the real axis.

3. The question of Boas and proof of Theorem 2. An entire function $f(z)$ of exponential type is to be defined for which

$$
\lim _{x \rightarrow \infty}|f(x+i y)|
$$

exists if and only if $y=0$. This function will be

$$
f(z)=z e^{-\pi z} \prod_{n=1}^{\infty}\left(1-\begin{array}{c}
z^{2} \\
\lambda_{n}^{2}
\end{array}\right)
$$

where

$$
\lambda_{n}={ }_{V}^{n} \exp \left(\begin{array}{cc}
i \pi & o_{n} \\
4 &
\end{array}\right)
$$

Here

$$
\hat{o}_{n}= \pm 1
$$

is to be determined. Pairs of zeros of $f(z)$ are replaced by their conjugates on changing the sign of $\partial_{n}$. Thus the modulus of $f(x)$ is independent of the choice of $\delta_{n}$, but $|f(x+i y)|$ does in general depend on $\delta_{n}$.

In case $\delta_{n}=1$ for all $n$ let the function be designated $f_{0}(z)$, that is, define

$$
f_{0}(z)=z e^{-\pi z} \prod_{1}^{\infty}\left(1-\frac{2 z^{2}}{i n^{2}}\right)=e^{-\pi z} \frac{\sin \pi(1-i) z}{\pi(1-i)} .
$$

Likewise, if $\delta=-1$ for all $n$ call the function $f_{1}(z)$,

$$
f_{1}(z)=z e^{-\pi z} \prod_{1}^{\infty}\left(1+\begin{array}{c}
2 z^{2} \\
i n^{2}
\end{array}\right)=e^{-\pi z \sin \pi(1+i) z} \pi(1+i) .
$$


Thus we have

$$
\begin{aligned}
& \lim _{x \rightarrow \infty}\left|f_{0}(x+i y)\right|=\begin{array}{c}
e^{-\pi \eta} \\
2 \pi V / 2
\end{array} \\
& \lim _{x \rightarrow \infty}\left|f_{1}(x+i y)\right|=\begin{array}{c}
e^{\pi \prime \prime} \\
2 \pi V / 2
\end{array}
\end{aligned}
$$

The $\partial_{n}$ are to be chosen so that in some neighborhoods $f(z)$ will have approximately the same modulus as $f_{0}(z)$, while in other neighborhoods $f(z)$ will have approximately the same modulus as $f_{l}(z)$.

Let $N_{1}, N_{i}, \cdots$ be a sequence of integers such that

$$
N_{\nu+1} \geq 4 N_{\nu}
$$

and choose $N_{1}=1$. Define

$$
\delta_{n}=(-1)^{2} \text {, }
$$$$
N_{\nu} \leqq n<N_{i+1} .
$$

This completes the definition of the function $f^{\prime}(z)$. If $z$ is even then for $z$ in the ring $N_{2}^{2} \leqq|z| \leqq 2 N_{\nu}^{2}$ we have

$$
\left|\varkappa \log \frac{f(z)}{f_{0}(z)}\right| \leqq \sum_{n<N_{\nu}}\left|\log \frac{1-\lambda_{n}^{2} / z^{2}}{1-n^{2} i / 2 z^{2}}\right|+\sum_{n>N_{\nu+1}}\left|\log \frac{1-z^{2} / \lambda_{n}^{2}}{1-2 z^{2} / i n^{2}}\right| .
$$

Using the estimates

$$
|\log (1-u)| \leqq|2| u \mid,
$$

and $\sum_{1}^{k} n^{2} \leqq k^{3}, \sum_{k}^{\infty} n^{-2}<2 / k$ we have

$$
\left|\oiiint \log \begin{array}{r}
f(z) \\
f_{0}(z)
\end{array}\right| \leq \begin{gathered}
18 \\
N_{\nu}
\end{gathered}, \quad \quad N_{\nu}^{*} \leq|z| \leq 2 N \%
$$

Likewise, if $\nu$ is odd we have

$$
\left|\% \log \begin{array}{c}
f(z) \\
f_{1}(z)
\end{array}\right| \leqq \begin{gathered}
18 \\
N_{\nu}
\end{gathered}, \quad \quad N_{\nu}^{z} \leq|z| \leq 2 N_{\nu}^{\prime} .
$$

These inequalities together with (27), (28) show that

$$
\limsup _{x \rightarrow \infty}|f(x+i y)|=\frac{e^{\pi|y|}}{2 \pi \sqrt{2}}
$$

and

$$
\liminf _{x \rightarrow \infty}|f(x+i y)|=\begin{gathered}
e^{-\pi|y|} \\
2 \pi V / 2
\end{gathered} .
$$

It is also clear that $f(z)$ is an entire function of exponential type. Indeed, 


$$
\left|f\left(r \cdot e^{i \theta}\right)\right| \leq r e^{\pi r} \prod_{n=1}^{\infty}\left(1+\begin{array}{c}
2 r^{*} \\
n^{2}
\end{array}\right)<e^{\pi(1+v \varepsilon) r} .
$$

We now turn to proof of Theorem 2. Thus suppose that f(z) satisfies the conditions of Theorem 2, so

$$
\mid f(z) \leqq A e^{\tau|z|}
$$

for some $A, \tau$. Since $|f(x)|$ has a finite limit it follows that the functions $f(z) e^{i \tau z}$ is bounded on the positive halves of the real and imaginary axes. Then the Phragmén-Lindelöf principal shows that since this function is of order one it is bounded throughout the first quadrant. Likewise, $f(z) e^{-i \tau z}$ is bounded in the fourth quadrant, and we have

$$
|f(x+i y)| \leqq B e^{\tau \mid y_{i}^{i}},
$$

write

$$
L=\lim _{x \rightarrow \infty}|f(x)|, \quad L_{m}=\lim _{r \rightarrow \infty}\left|f^{(m)}(x)\right| .
$$

Now let $a_{1}, a_{2}, a_{3}, \cdots$ be any sequence of real numbers such that $a_{n} \rightarrow \infty$ as $n \rightarrow \infty$. Because of (29) the functions $f\left(z+a_{n}\right)$ are uniformly bounded in each bounded domain for large $n$. Thus there is a subsequence $b_{1}, b_{2}, b_{3}, \cdots$ of the $a_{n}$ such that the functions $f\left(z+b_{n}\right)$ tend to a limit $F(z)$ as $n$ becomes large,

$$
F(z)=\lim _{n \rightarrow \infty} f\left(z+b_{n}\right) .
$$

Then $F(z)$ is an entire function of exponential type, indeed it satisfies

$$
|F(x+i y)| \leqq B e^{\tau|y|}
$$

for all real $x, y$. We also have

$$
F^{(\nu)}(z)=\lim _{n \rightarrow \infty} f^{(\nu)}\left(z+b_{n}\right), \quad \nu=0,1,2, \cdots
$$

The theorem will follow if it is shown that the value of $\left|F^{(\nu)}(z)\right|$ is a function of $y$ only and is independent of the particular sequence $a_{1}, a_{2}, \cdots$.

Now $F(z)$ has the constant modulus $L$ on the real axis. If $L=0$ then $F(z)$ and all its derivatives vanish identically so $(9)$ is true. Thus suppose $L \neq 0$. Then $F(z)$ can have no zero, for it would then have a pole at the conjugate point. Thus

$$
F(z)=L e^{i \gamma} e^{i \alpha}
$$

where $\gamma, c$ are real numbers. Now

$$
F^{(m)}(z)=L e^{i \gamma}(i \alpha)^{m} e^{\prime \alpha z},
$$


and $F^{(m)}(z)$ has the constant modulus $L_{m}$ on the real axis. This gives the relation

$$
\alpha= \pm\left(L_{m} / L\right)^{1 / m} .
$$

If $L_{m}=0$ then $\alpha=0$ for all sequences and (9) is true. Thus suppose $L_{m}>0$. Now consider the values of $|f(x+i)|$. If one sequence $a_{1}, a_{2}, \cdots$ leads to a function $F(z)$ of the form (30) with $\alpha=\left(L_{m} / L\right)^{1 / m}$, and another sequence leads to a function with $\alpha=-\left(L_{m} / L\right)^{1 / m}$ then

$$
\limsup _{x \rightarrow \infty}|f(x+i)|=L e^{|a|}, \quad \liminf _{x \rightarrow \infty}|f(x+i)|=L e^{-|\alpha|} .
$$

Now $|f(x+i)|$ varies continuously as $x$ increases, so there will be a sequence $x_{1}, x_{2}, \ldots$ with $x_{n} \rightarrow \infty$ as $n \rightarrow \infty$ such that $\left|f\left(x_{n}+i\right)\right|=L$. A subsequence of the functions $f\left(z+x_{n}\right)$ will tend to a limit $F(z)$ satisfying $|F(i)|=L$. But $F(z)$ will be of the form (30), so $\alpha=0$. This contradicts (31) and proves the theorem.

Indeed, the forgoing argument proves slightly more than the theorem states. First, under the conditions of Theorem 2 we have

$$
\lim _{x \rightarrow \infty}\left|f^{(\nu)}(x+i y)\right|=L|\alpha|^{\nu} e^{-\alpha y}, \quad y=0,1,2, \cdots,
$$

where $L, \alpha$ are independent of $\nu, y$. Secondly, the requirement that $f(z)$ is an entire function of exponential type can be replaced by the supposition that $f(z)$ is regular in some half-plane $x \geq c$, where it is of exponential growth.

\section{REH ERENCES}

1. S. Agmon, Functions of exponential type in an angle and singularitics of Taylor. scries, Tans Amer. Math. Soc., 70 (1951), 492-508.

2. R. P. Boas, Jr., Entire Functions, Academic Press, New York.

3. Bull. Amer. Math. Soc., 60 (1954), 97.

4. R. J. Duffin and A. C. Schaeffer, Power scrics wilh bounded coefficionts, Amer. J. Math., 67 (1945), 141-154.

UNIVERSITY OF WISCONSIN 


\section{PACIFIC JOURNAL OF MATHEMATICS}

EDITORS

\author{
H. L. Royden \\ Stanford University \\ Stanford, California \\ E. Hewite \\ University of Washington \\ Seattle 5 , Washington
}

\author{
R. P. Dilworth \\ California Institute of Technology \\ Pasadena 4, California
}

\author{
A. Horn* \\ University of California \\ Los Angeles 24, California
}

\section{ASSOCIATE EDITORS}
E. F. BECKENBACH
M. HALL
M. S. KNEBELMAN
J. J. STOKER
C. E. BURGESS
P. R. HALMOS
I. NIVEN
V. GANAPATHY IYER
T. G. OSTROM
G. SZEKERES
H. BUSEMANN
R. D. JAMES
M. M. SCHIFFER
F. WOLF
H. FEDERER

\section{SPONSORS}

\author{
UNIVERSITY OF BRITISH COLUMBIA \\ CALIFORNIA INSTITUTE OF TECHNOLOGY \\ UNIVERSITY OF CALIFORNIA \\ CALIFORNIA RESEARCH CORPORATION \\ MONTANA STATE UNIVERSITY \\ UNIVERSITY OF NEVADA \\ OREGON STATE COLLEGE \\ UNIVERSITY OF OREGON \\ UNIVERSITY OF SOUTHERN CALIFORNIA
}

\author{
STANFORD UNIVERSITY \\ UNIVERSITY OF UTAH \\ WASHINGTON STATE COLLEGE \\ UNIVERSITY OF WASHINGTON \\ AMERICAN MATHEMATICAL SOCIETY \\ HUGHES AIRCRAFT COMPANY
}

Mathematical papers intended for publication in the Pacific Journal of Mathematics should be typewritten (double spaced), and the author should keep a complete copy. Manuscripts may be sent to any of the editors. Manuscripts intended for the outgoing editors should be sent to their successors. All other communications to the editors should be addressed to the managing editor, Alfred Horn at the University of California, Los Angeles 24, California.

50 reprints of each article are furnished free of charge; additional copies may be obtained at cost in multiples of 50 .

The Pacific Journal of Mathematics is published quarterly, in March, June, September, and December. The price per volume (4 numbers) is $\$ 12.00$; single issues, $\$ 3.50$. Back numbers are available. Special price to individual faculty members of supporting institutions and to individual members of the American Mathematical Society: $\$ 4.00$ per volume; single issues, $\$ 1.25$.

Subscriptions, orders for back numbers, and changes of address should be sent to Pacific Journal of Mathematics, c/o University of California Press, Berkeley 4, California.

Printed at Kokusai Bunken Insatsusha (International Academic Printing Co., Ltd.), No. 10, 1-chome, Fujimi-cho, Chiyoda-ku, Tokyo, Japan.

* During the absence of E. G. Straus.

PUBLISHED BY PACIFIC JOURNAL OF MATHEMATICS, A NON-PROFIT CORPORATION COPYRIGHT 1956 BY PACIFIC JOURNAL OF MATHEMATICS 


\section{Pacific Journal of Mathematics}

\section{Vol. 6, No. $2 \quad$ December, 1956}

Louis Auslander, Remark on the use of forms in variational calculations .......................................... 209

Hubert Spence Butts, Jr. and Henry B. Mann, Corresponding residue systems in algebraic number fields ........................ 211

L. Carlitz and John Herbert Hodges, Distribution of matrices in a finite field............................................

Paul Civin and Bertram Yood, Invariant functionals ............... 231

David James Dickinson, Henry Pollak and G. H. Wannier, On a class of polynomials orthogonal over a denumerable set .................

Bernard Friedman and Luna Mishoe, Eigenfunction expansions associated with a non-self-adjoint differential equation ....................

Luna Mishoe and G. C. Ford, On the uniform convergence of a certain eigenfunction series .............................. 271

John W. Green, Mean values of harmonic functions on homothetic curves...........................................

Charles John August Halberg, Jr. and Angus E. Taylor, On the spectra of linked operators .....................................

Chuan Chih Hsiung, Some integral formulas for closed hypersurfaces in Riemannian space ................................... 291

Norman D. Lane, Differentiable points of arcs in conformal n-space 301

Louis F. McAuley, A relation between perfect separability, completeness, and normality in semi-metric spaces

G. Power and D. L. Scott-Hutton, The slow shearing motion of a liquid past a semi-infinite plane .............................

A. C. Schaeffer, Entire functions

Edward Silverman, An intrinsic inequality for Lebesgue area...

Choy-Tak Taam, Asymptotic relations between systems of differential equations.

Ti Yen, Quotient algebra of a finite $A W^{*}$-algebra ... 